\title{
Wohin führt die kausale Handlungstheorie?
}

\author{
Georg Gasser, Innsbruck
}

Wer der Frage nachgeht, was jemand tut, der geht - wenigstens implizit auch der Frage nach, wer da etwas tut. Handlungen schreiben wir normalerweise Personen zu. Handeln zu können ist eine derjenigen Eigenschaften, die Personen auszeichnet. Die Analyse menschlichen Handelns geht mit der Frage einher, was menschliche Personen sind. Folgender Beitrag befasst sich mit dem Verhältnis von Handlung und Person innerhalb der kausalen Theorie des Handelns. Durch eine eingehende Analyse dieses weitverbreiteten Ansatzes zur Erklärung und Deutung menschlichen Handelns sollen Implikationen für den Begriff des Handelnden herausgearbeitet werden.

\section{Die kausale Theorie des Handelns}

\subsection{Gründe sind Ursachen}

Die aktuelle Debatte der Handlungstheorie innerhalb der analytischen Philosophie ist von der kausalen Theorie des Handelns geprägt. Diese kann geradezu als ihre Orthodoxie bezeichnet werden. ${ }^{1}$ Die Grundthese der kausalen Handlungstheorie besagt, dass ein Ereignis dann als Handlung gelten kann, wenn dieses Ereignis (i) durch die Gründe des Handelnden verursacht wird, und zwar (ii) in der richtigen Art und Weise. „In der richtigen Art und Weise" besagt, dass die Gründe jene Rolle in der Generierung der Handlung spielen müssen, welche ihnen vom Handelnden zugesprochen wird. Stellen wir uns vor, Josef beabsichtigt, seine Tante zu überfahren. Der Gedanke an diese Tat lässt ihn so unaufmerksam werden, dass er eine Dame auf dem Zebrastreifen übersieht und überfährt, die zufälligerweise seine Tante ist. In einem solchen Fall liegt zwar ein Grund und die dem Grund entsprechende Handlung vor, aber sie sind nicht ,in der richtigen Weise“ kausal miteinander verknüpft: Das Überfahren von

1 Siehe dazu Keil (2000), 18.

Niederbacher, B. / Runggaldier, E., Hrsg. (2008), Was sind menschliche Personen? Ein akttheoretischer Zugang. Ontos verlag, 181-208. 
Josefs Tante war nämlich eine unmittelbare Folge von Josefs Unaufmerksamkeit und nicht die unmittelbare Folge von Josefs Absicht, seine Tante zu überfahren.

Dieses Beispiel macht die argumentative Kraft der kausalen Theorie des Handelns deutlich: Wenn Gründe nicht „,in der richtigen Art und Weise“ für die Generierung einer Handlung verantwortlich sind, so bleibt unklar, wie Gründe für das Auftreten einer Handlung relevant sein können. Nur wenn Gründe ,in der richtigen Art und Weise“ eine Handlung generieren, können diese Gründe auch als entscheidende Faktoren für das Hervorbringen der entsprechenden Handlung angesehen werden. ${ }^{2}$

Anders ausgedrückt: Wenn zwischen Grund und Handlung keine Kausalbeziehung ,in der richtigen Art und Weise“ vorliegt, dann kann nicht zwischen einem möglichen, aber unwirksamen Handlungsgrund und dem tatsächlich wirksamen Handlungsgrund unterschieden werden. Oft lassen sich nämlich viele gute Gründe für das Eintreten einer Handlung angeben, aber die Wirksamkeit eines Grundes lässt sich nicht daran ablesen, ob ein Grund gut oder weniger gut ist. Es ist ja durchaus möglich, dass ein Verhalten, für das es gute Gründe gibt, durch etwas anderes als diese Gründe verursacht worden ist. Wie die Geschichte von Josef und seiner Tante illustriert, waren Gründe für die Ermordung der Tante zwar in Josef hochgekommen, aber sie haben nicht ,in der richtigen Art und Weise" Eingang in den kausalen Prozess gefunden, welcher zum geplanten Resultat - dem Tod der Tante - führte. Daher, so lautet die Schlussfolgerung der kausalen Theorie des Handelns, muss der Grund für die Handlung auch die Ursache dieser Handlung sein. Sonst könnte ein angegebener Grund zwar verständlich machen, warum diese Handlung vollzogen wurde - der angegebene Grund ,passt“ zur stattgefundenen Handlung: Josefs Mordgedanke könnte verständlich machen, warum Josef seine Tante überfahren hat. Aber besagter Grund würde das Eintreten der Handlung nicht erklären, da die Handlung ja nicht wegen dieses Grundes, sondern wegen etwas anderem stattgefunden hat.

2 Mele (1992),7, bringt diese Überlegung folgendermaßen auf den Punkt: “(...) it is difficult to see how a reason can account for someone's A-ing if it (or the agent's having it) does not play a suitable role in the etiology of his A-ing." 
Die Deutung von Gründen als Ursachen erweist sich somit als sehr attraktiv: Einerseits ermöglicht der Verweis auf den Handlungsgrund eine rationale Beurteilung der Handlung. Wir können danach fragen, ob die Gründe, die ausschlaggebend für den Vollzug der Handlung waren, gute oder schlechte bzw. angebrachte oder unangebrachte Gründe waren. Andererseits können Handlungen genau so wie alle übrigen Vorgänge der Wirklichkeit auch als Ursache-Wirkungs-Beziehungen konzipiert werden. Handlungen ,scheren nicht aus“ und bilden nicht eine von kausalen Vorgängen separate Klasse, bei der unklar ist, wie das Verhältnis zwischen Gründen und Handlungen $\mathrm{zu}$ bestimmen ist. Weiters spricht für eine kausale Bestimmung des Verhältnisses zwischen Gründen und Handlungen, dass Handlungen sich im Normalfall in Körperbewegungen manifestieren. Für Körperbewegungen lassen sich Ursachen neurophysiologischer Art angeben. Daher ist es plausibel anzunehmen, dass diese Körperbewegungen durch Ursachen hervorgerufen werden, die neurophysiologisch realisiert sind und Gründe für Handlungen folglich auf irgendeine Weise mit den Ursachen dieser Körperbewegungen identisch sein müssen.

\subsection{Die Redeweise von Gründen}

Die Behauptung, dass Gründe mit Ursachen identisch sind, ist laut kausaler Theorie des Handelns aber kein Anlass, nicht strikt zwischen der Redeweise von Gründen und Handlungen einerseits und der Redeweise von Ursachen und Wirkungen andererseits zu unterscheiden. Davidson, einer der profiliertesten Vertreter der kausalen Theorie des Handelns, betont ausdrücklich, dass die Rede von Gründen und die Rede von Ursachen zwei verschiedenen und nicht übersetzbaren Beschreibungsweisen der Wirklichkeit angehören. Im Gegensatz zu Beschreibungen im Bereich des Physischen verfügen wir bei Beschreibungen im Bereich des Psychischen nicht über gesetzesartige Zusammenhänge. Wenn wir auch von Regelmäßigkeiten im mentalen Bereich nach der Art ,wenn X beleidigt wird, dann wird X in der Regel zornig“ sprechen können, so sind diese Regelmäßigkeiten nicht zu 
verwechseln mit Gesetzmäßigkeiten, wie sie typischerweise im physischen Bereich auftreten. ${ }^{3}$

Da sich keine gesetzesartigen Zusammenhänge im Bereich des Mentalen angeben lassen, ist eine Rückführung des Mentalen auf Ursache-WirkungsProzesse, wie sie in der Neurophysiologie oder gar Physik beschrieben werden, laut Davidson unmöglich. Wollte man die postulierte Kausalbeziehung zwischen Grund und Handlung in der Sprache der Naturwissenschaften beschreiben, so käme man zu einer völlig anderen Art der Beschreibung: Nicht mehr von Handlungsgründen und von Handlungen wäre dann die Rede, sondern von neurologischen, chemischen oder physischen Ereignissen, die Kausalbeziehungen zueinander aufweisen. ${ }^{4}$

Daher ist im Fall menschlichen Handelns die Verwendung eines mentalintentionalen und nicht eines physischen Vokabulars nicht nur berechtigt, sondern sogar gefordert. Die Verwendung dieses intentionalen Vokabulars setzt zwar voraus, dass physische Mechanismen ablaufen, die den Überlegungen und entsprechenden Handlungen zugrunde liegen, aber diese Mechanismen müssen nicht angegeben werden können:

Knowledge of the rational abilities of an agent can sustain our confidence that a certain behavioural effect will show up, even if we do not have any knowledge of mechanisms, i.e. of the ways the organism produces this effect. To know that someone has certain beliefs and desires is to have a certain amount of causal information about him; but this sort of causal knowledge is not knowledge of mechanisms or of executing processes. ${ }^{5}$

Die Gründe eines Handelnden $\mathrm{zu}$ kennen, bedeutet folglich, eine Informationen über kausale Abläufe zu besitzen - auch wenn diese Information relativ offen und wage ist, da durch sie über die spezifische Art und Weise, wie die Handlung hervorgebracht wird, ja nichts ausgesagt wird.

3 Davidson (1993), 312, schreibt etwa: "What then are we 'ignorant' about when it comes to explaining psychological events? We don't know precise laws for explaining and predicting them; but unlike the situation in natural sciences, this isn't because we haven't discovered them yet; it's because there are no such laws."

4 Davidson $\left(1963 /^{2} 1982\right), 17$.

5 Lanz (1993), 300. 


\section{3. „Epistemisch-semantischer Dualismus, ontologischer Monismus“}

Davidson versucht aufzuzeigen, dass für das Verstehen von Handlungen als Vollzüge rational überlegender Personen der Begriffsapparat der Gründe, Überzeugungen und Absichten zentral ist. Ohne diesen Begriffsapparat könnte menschliches Handeln nicht verstanden werden. Auf die Ontologie hat die Verwendung dieses Begriffapparats laut Davidson aber keine Auswirkungen. Der Bereich des Mentalen stellt nämlich nicht eine ontologische, sondern eine rein begriffliche Kategorie dar. ${ }^{6}$ Die Redeweise vom Bereich des Mentalen bezieht sich nicht auf etwas, was es ,in der Wirklichkeit“ als „Mentales“ gibt. Mentale Vorgänge werden als „mentale“ beschrieben, sind ontologisch gesprochen aber physische Vorgänge: Gründe sind deshalb kausal wirksam, weil sie physische Ereignisse sind. Evnine führt deutlich aus, dass Davidson mentalen Eigenschaften kausale Relevanz abspricht - im Gegensatz zu physischen Eigenschaften:

They cannot cause what they cause because they have the content they do, since all their causally relevant properties are physical. This in itself does not mean mental events cannot be causes of actions. But it does mean that there is no connection between their having what causal powers they do and their having what propositional content they do. ${ }^{7}$

Peter Lanz bestätigt diese Leseart der Davidson'schen Position:

To see rational causes at work does not commit one to a non-physicalist ontology, bur rather reflects the cognitive needs of rational agents in their interactions among one another. ${ }^{8}$

Die Redeweise von Gründen hat primär die kognitiven Bedürfnisse von uns Handelnden im Blick. Diese kognitiven Bedürfnisse können nicht in eine Sprache der Ursachen und Wirkungen übersetzt bzw. durch eine solche befriedigt werden. Die Redeweise von Gründen ist sozusagen unhintergehbar, um unser Selbstverständnis als handelnde und soziale Wesen aufrechterhalten zu können. ${ }^{9}$

\footnotetext{
So Davidson (1987/2006), 199-200.

Evnine (1991), 161.

Lanz (1993), 300.

9 Davidson betont diese Tatsache ausdrücklich, z.B. in Davidson (1987/2006), 200201: „Durch Grund-Erklärungen werden andere Personen für uns nur in dem Maße verständlich, in dem wir etwas von der Art unserer eigenen Denkkräfte am Werk
} 
Diese Einsicht ist zweifelsohne richtig. Der Eindruck drängt sich aber trotzdem auf, dass diese Argumentation primär als Zugeständnis an unser „psychologisches make-up“ zu werten ist: Der menschliche Lebensvollzug ist dergestalt, dass wir ohne Begrifflichkeiten wie Gründe, Wünsche und Handlungen nicht auskommen. Würden wir sie durch die Begrifflichkeiten der Ursachen, Wirkungen und Kausalbeziehungen ersetzen, so würden unsere lebensweltlichen Vollzüge zusammenbrechen. Wir wären nicht mehr fähig, uns als rational handelnde Wesen zu begreifen. Wie auch immer in concreto die Argumentation für die Unhintergehbarkeit der Sprechweise vom mentalen Bereich gelagert ist, spielt an diesem Punkt keine wesentliche Rolle. Entscheidend ist vielmehr, dass der kausalen Theorie des Handelns zufolge der Unterschied zwischen den Begrifflichkeiten der Gründe und Handlungen und den Begrifflichkeiten der Ursachen und Wirkungen darin besteht, dass letztere Begrifflichkeit dem, was es eigentlich gibt, näher zu stehen bzw. zu entsprechen scheint. Ihr fällt nämlich nicht nur eine erkenntnistheoretische und semantische, sondern auch eine ontologische Relevanz zu. Diese Davidson'sche Explikation der kausalen Theorie des Handelns erinnert stark an jene Position, die Habermas unter dem etwas schwammigen Schlagwort „epistemischer Dualismus, ontologischer Monismus ${ }^{\text {“10 }}$ zusammenfasst.

\subsection{Weiterführungen dieser Position}

Im Anschluss an diese Position stellt sich die Frage, wie sich die Identitätsbehauptung zwischen Gründen und Ursachen zum vorgeschlagenen semantisch-epistemischen Dualismus verhält: Lässt sich der epistemische und semantische Dualismus angesichts der ontologischen Vorrangstellung des physischen Bereichs aufrechterhalten? Oder muss es letzten Endes doch zu einer Umdeutung menschlichen Handelns in eine bestimmte Art physischer Vorgänge kommen?

Ein Fundort, diesen Fragen nachzugehen, ist Searles Theorie der intentionalen Verursachung. Für Searle gibt es in Bezug auf Handlungen

erkennen können. Es wäre ein Irrtum, wollte man annehmen, dies sei lediglich ein Zeichen mangelnder Phantasie oder vielleicht mangelnder Sympathie. Es ist ein zentrales und unersetzliches Merkmal des Intentionalen.“ 
zwar zwei mögliche Beschreibungsweisen des vorliegenden „Tatbestands“, aber er warnt davor, aus der Möglichkeit dieser zweifachen Beschreibung große philosophische Schlüsse zu ziehen. Wie Davidson lehnt er eine dualistische Deutung mentaler Zustände ab. Klarer als Davidson macht sich Searle aber gegen eine Favorisierung der physisch-kausalen Beschreibungsweise gegenüber der mental-intentionalen stark.

Für Searle drückt die mental-intentionale Beschreibung genauso wie die physisch-kausale Beschreibung den vorliegenden Tatbestand aus: Wenn wir die intentionale Beziehung zwischen Gründen und Handlungen als unumgänglichen Bestandteil unseres kognitiven Apparats auffassen, so sollten wir diese intentionale Beziehung nicht nur kognitiv, sondern auch ontologisch ernst nehmen. Die Unterscheidung zwischen „mental“ und „physisch“ ist mit sehr großer Vorsicht zu genießen. ${ }^{11}$ In der Philosophie führe sie nämlich zur ,outmoded dualistic/materialistic assumption that the "mental" character of consciousness makes it impossible for it to be a "physical” property. “12

Für die Handlungstheorie bedeutet dies, dass bei Handlungen unabhängig von entsprechenden Beschreibungen ein logisch-begrifflicher Zusammenhang zwischen Ursache und Wirkung besteht, da die Ursache die Wirkung repräsentiert bzw. die Wirkung eine Repräsentation der Ursache ist. Searle verdeutlicht diese Behauptung anhand des Zustands, durstig zu sein: Durstig zu sein geht mit dem Wunsch zu trinken einher. Zu den Erfüllungsbedingungen dieses Wunsches gehört es zu trinken. Wer also im Zustand ist, durstig zu sein, der verspürt den Wunsch zu trinken und dieser Wunsch ist dann erfüllt, wenn man trinkt. Der Wunsch zu trinken repräsentiert die Handlung des Trinkens als seine Erfüllungsbedingung. Diese Repräsentation hängt kausal mit den Erfüllungsbedingungen des entsprechenden Wunsches dahingehend zusammen, dass es zu den Erfüllungsbedingungen des Wunsches gehört, sie herbeizuführen. Mit anderen Worten: Zwischen Durst und Trinken besteht ein intentionaler und kausaler Zusammenhang dahingehend, dass es zum intentionalen Gehalt des Zustandes Durst gehört, trinken zu wollen,

11 Siehe Searle (1992), Kap. 4.

12 Searle (1992), 91. 
und dieser Zustand Durst die Handlung trinken als seine Erfüllungsbedingung kausal auslöst. Searle schreibt:

Die Angabe von Ursache und Wirkung unter diesen kausal relevanten Aspekten wird uns - da ja Intentionalität und die Erfüllungsbedingungen dieser Intentionalität zu ihr gehören - logisch zusammenhängende Beschreibungen von Ursache und Wirkung gerade deshalb liefern, weil Ursache und Wirkung selbst logisch zusammenhängen. Sie sind nicht durch die Folgerungsbeziehung logisch verbunden, sondern vielmehr durch den intentionalen Gehalt und Erfüllungsbedingungen. Ich glaube, es zeugt von einer grundlegenden Verwirrung, wenn wir annehmen, dass Ereignisse nur unter einer Beschreibung logisch zusammenhängen können, denn Ereignisse selbst können intentionale Beziehungen haben, die sie logisch zueinander in Beziehung setzen, gleichgültig, wie sie beschrieben werden. ${ }^{13}$

Ontologisch gesehen sind Gründe spezielle Zustände bzw. Ereignisse im Gehirn, die dank ihres intentionalen Gehalts als Wirkungen die Erfüllungsbedingungen dieser Gehalte hervorrufen können. Diese Wirkungen sind im Normalfall Körperbewegungen, durch welche die Handlung ausgeführt wird. Searles Theorie der intentionalen Verursachung besagt somit folgendes: Intentionale Zustände sind ein bestimmter Typ neurophysiologischer Zustände, welche sich dadurch auszeichnen, dass sie die Erfüllungsbedingungen ihres intentionalen Gehalts repräsentieren und durch diese Repräsentation in Form von Körperbewegungen verursachen. Angewandt auf das obige Beispiel des Durstigseins: Der Zustand, durstig zu sein, geht mit der Absicht zu trinken einher. Der Gehalt dieser Absicht repräsentiert nicht nur seine Erfüllungsbedingungen, nämlich den Durst durch Trinken zu löschen, sondern diese Repräsentation verursacht zugleich die Körperbewegungen, welche das Löschen des Durstes durch Trinken realisieren. Handlungen bestehen aus einem „mentalen“ und einem ,physischen“ Bestandteil, wobei der „mentale“ Bestandteil den physischen Bestandteil intentional bereits repräsentiert und als seine Erfüllungsbedingung verursacht. ${ }^{14}$

Searles Ansicht, die Rede vom „Mentalen“ und „Physischen“ entstamme einem nicht mehr zeitgemäßen Modell des Bewusstseins, führt

13 Searle (1987), 157.

14 Siehe in diesem Zusammenhang die skizzenhafte Zusammenfassung der Theorie der intentionalen Verursachung durch Searle selbst in Lepore/Van Gulick (1991), $295 \mathrm{ff}$. 
dazu anzunehmen, dass es eigentlich nur mehr den physischen bzw. biologischen Bereich gibt. Innerhalb dieses Bereichs gibt es dann der Art nach verschiedene Vorgänge, u.a. eben auch Vorgänge, die ihre Erfüllungsbedingungen repräsentieren und intentional verursachen können. Inwieweit eine solche Redeweise einen Vorteil gegenüber der Unterscheidung zwischen ,physisch“ und „mental“ darstellt, lasse ich dahingestellt. Zweifelsohne lässt sich aus Searles Ausführungen aber herauslesen, dass Handlungen eine von anderen ,natürlichen“ Abläufen verschiedene Struktur haben. Andere „natürliche“ Abläufe lassen sich hinreichend als Abläufe auffassen, die aus Ereignissen bestehen, welche kausal miteinander in Beziehung stehen. Im Fall des menschlichen Verhaltens hat die Ursache hingegen einen Gehalt, und die Erklärung menschlichen Verhaltens ist nur dann eine gültige Erklärung, wenn sie den Gehalt der Ursache berücksichtigt. Ursachen natürlicher Abläufe unterscheiden sich von Handlungsursachen dahingehend, dass nur letztere einen Gehalt haben und dieser Gehalt wesentlich festlegt, was als Folge dieser Handlungsursache gilt und was nicht.

Searle geht insofern über Davidson hinaus, als er dafür argumentiert, die Rede von mentalen Zuständen nicht der Rede von physischen Zuständen gegenüberstellen zu wollen. Während bei Davidson die beiden Redeweisen denselben vorliegenden Tatbestand jeweils auf verschiedene Weise beschreiben und erklären, etwa in Form physischer Vorgänge oder in Form von Gründen und Handlungen, wendet Searle gegen eine solche Unterscheidung ein, dass sie den vorliegenden Tatbestand eher verdecke als erläutere. Die Redeweise von Ursache und Wirkung und die Redeweise von Grund und Handlung fallen beim menschlichen Handeln zusammen, da Ursachen von Handlungen intentionale Ursachen sind und Handlungen als ihre Wirkungen vom intentionalen Gehalt verursacht werden. Handlungen sind nicht nur Ereignisketten, die dank entsprechender Beschreibungen in ein intentionales Gewand gekleidet werden, sondern bestimmte Ereignisketten weisen vielmehr „Intentionalität“ als ihren spezifischen „,biologischen Bestandteil“ auf, so wie andere Ereignisketten die Verdauung oder Photosynthese. ${ }^{15}$

15 Searle (1992), 93. 
Searles Ausführungen sind vielfach unscharf und bedürften der Erläuterung. Kann man Searles Annahmen konkreter ausformulieren? Was heißt es, dass mentale Bestandteile einer Handlung nichts anderes als biologische Zustände mit intentionalem Gehalt sind, die durch diesen intentionalen Gehalt ihre Erfüllungsbedingungen verursachen?

Mir scheint, eine mögliche Konkretisierung der Searle'schen Auffassung ist bei Ansgar Beckermann zu finden: Beckermann verweist zu Beginn seiner Argumentation darauf, dass das Feuern von Neuronen kognitiven Gehalt haben kann. Als Beispiel hierfür zieht er die Entdeckung der sogenannten Kantendetektoren heran: Es handelt sich um Neuronenverbände, deren Feuerungsrate stark ansteigt, wenn im Gesichtsfeld der entsprechenden Person eine Kante mit einer gewissen Orientierung liegt. Ähnliche Neuronenverbände wurden anscheinend im Zusammenhang mit der Wahrnehmung von Mimik und Gestik entdeckt. ${ }^{16}$ Ausgehend von diesen Überlegungen zieht Beckermann den Schluss, dass es sich bei bestimmten neuronalen Prozessen auch um Prozesse des rationalen Überlegens handelt. So wie bestimmte Neuronenverbände in besonderer Weise für das Wahrnehmen von Oberflächenstrukturen oder von Gesichtern zuständig sind, so reagieren andere Neuronenverbände besonders sensitiv auf Argumente und rationale Überlegungen. Die Aktivität dieser auf Bedeutungen sensitiv reagierenden Neuronenverbände ist folglich als Ursache für eine Handlung zu deuten, welche als Reaktion auf die zuvor neuronal verarbeiteten Argumente zu sehen ist. Da diese spezifischen neuronalen Prozesse intentionale Gehalte verarbeiten können, sind sie auch fähig, angemessene Antworten auf diese Gehalte zu verursachen. ${ }^{17}$ Angewandt auf Searles Überlegungen bedeutet dies: Verläuft die Verarbeitung der (re-)präsentierten Gehalte durch die Neuronenprozesse erfolgreich, so verursacht diese Verarbeitung Körperbewegungen, welche die Erfüllungsbedingungen der (re-) präsentierten Gehalte darstellen und somit zur geplanten Wirkung - der gelungenen Handlung - führen.

16 Beckermann (2006), 302.
17 Beckermann (2006), 303. 
Von Davidson über Searle zu Beckermann führt keine geradlinige Entwicklungslinie. Alle drei Autoren fühlen sich der kausalen Theorie des Handelns verpflichtet und stellen ein bestimmtes Spektrum von verschiedenen Ausformulierungen dieser Theorie dar. Die Diskussion ihrer Ansätze sollte deutlich gemacht haben, dass in der kausalen Theorie des Handelns beim epistemisch-semantischen Beschreibungsdualismus à la Davidson häufig nicht halt gemacht wird. Wir haben gesehen, dass selbst Davidson diesen Beschreibungsdualismus nicht klar durchhält, sondern physische Beschreibungen im Gegensatz zu mentalen auch ontologisch ernst nimmt. Searle und Beckermann gehen noch einen Schritt weiter und fragen im Gegensatz zu Davidson ausdrücklich danach, wie Ursachen Gründe sein können bzw. wie eine Realisierung von Gründen in physisch beschreibbaren Ursache-Wirkungs-Relationen ausschauen könnte. Es geht ihnen um die Frage, welche Beschaffenheiten jene Entitäten aufweisen, die als Gründe Ursachen sind.

Searles Versuch, Davidsons Beschreibungsdualismus durch die Theorie der intentionalen Verursachung aufzubrechen und Beckermanns Versuch, die Gründe-sind-Ursachen-These neurowissenschaftlich zu untermauern, weisen darauf hin, dass für etliche Sympathisanten der kausalen Theorie des Handelns die Frage, wie Gründe Ursachen sein können, nicht übergangen werden darf.

\section{Probleme der kausalen Theorie des Handelns}

Wenden wir uns nach der Darstellung der kausalen Theorie des Handelns ihrer Bewertung zu. Kern der kausalen Theorie des Handelns ist die These, dass Gründe Ursachen sind. Aus dieser These ergibt sich die Forderung, Gründe ereignishaft zu deuten, da Ursachen normalerweise als Ereignisse aufgefasst werden. Im folgenden Abschnitt soll der Frage nachgegangen werden, was es heißt, dass Gründe qua Ursachen als Ereignisse aufzufassen sind. Ich werde in diesem Zusammenhang drei mögliche Problembereiche ansprechen, die mit dieser Identitätsthese einherzugehen scheinen: (i) Die Frage, ob der Begriff des Grundes einer Handlung (reason for action) nicht auf unklare Weise verwendet wird; (ii) die Frage, 
ob die kausale Theorie des Handelns klar zwischen wirksamen und nichtwirksamen Gründen unterscheiden kann und (iii) die Frage, ob in der kausalen Theorie des Handelns nicht eine starke Tendenz weg von personalen hin $\mathrm{zu}$ sub- und a-personalen Erklärungsmustern von Handlungen besteht.

\subsection{Gründe, Ursachen und Ereignisse}

Eine entscheidende Frage für den Vertreter der kausalen Theorie des Handelns ist, wie sich Gründe in die Kategorie der Ereignisse einordnen lassen. Was als Grund in Frage kommt, steht nicht von vornherein fest. Vielmehr ist es der Handelnde, der aus einer Vielzahl ihm zugänglicher Gründe einige herausgreift und $\mathrm{zu}$ seinen Gründen macht. Indem ein möglicher Grund für eine Handlung zum Grund des Handelnden wird, wird das Verhalten des Handelnden prinzipiell versteh- und nachvollziehbar. Die Handlung wird im Lichte des Handlungsgrundes für andere Personen zugänglich. Peter Hacker weist auf diese Funktion von Gründen in der Erklärung menschlichen Handelns ausdrücklich hin.

In saying that he is $\mathrm{V}$-ing because it is the case that $\mathrm{R}, \mathrm{A}$ is not merely citing the fact that $\mathrm{R}$ is a reason for him to $\mathrm{V}$; he is declaring it to be his reason for V-ing, endorsing a particular teleological explanation of his $\mathrm{V}$-ing, and taking responsibility for it under the description ' $\mathrm{V}$-ing, for the reason that $\mathrm{R}$ '. ${ }^{18}$

Falls Hackers Ausführungen korrekt sind, so besteht die primäre Funktion von Gründen darin, eine Handlung durch eine bestimmte Sichtweise, nämlich der des Handelnden, einer Erklärung zuzuführen. Mithilfe von Gründen können wir überhaupt erst verstehen, welche Erklärungen für die zur Debatte stehende Handlung herangezogen werden können.

Ist es aber sinnvoll zu sagen, dass eine kausale Beziehung zwischen Handlungsgrund und Handlung hergestellt wird, wenn der Handelnde aus der Vielzahl möglicher Gründe einen Grund herausgreift und dieser Grund zum Handlungsgrund wird? Es ist schwierig zu sehen, wie Gründe in die Kategorie der Ereignisse eingeordnet werden können, da der wesentliche Aspekt eines Grundes sein intentionaler Gehalt ist. Dank dieses Gehalts kann eine Handlung überhaupt erst gedeutet und einem Handelnden zugeschrieben werden. Vertreter der kausalen Theorie des Handelns sind

18 Hacker (2007), 223. 
sich des Unterschieds zwischen Gründen i.S. intentionaler Gehalte und Ursachen i.S. von Abläufen durchaus bewusst: Gründe eignen sich nicht als Ursachen - wenigstens nicht unmittelbar -, da ihnen ein ereignishafter Charakter abgeht. Daher wird oftmals auf die Änderungen intentionaler Einstellungen bzw. auf das Auftauchen neuer Gründe als die eigentlichen Gründe des Handelns verwiesen, da Veränderungen sehr wohl als Ereignisse aufgefasst werden können. In Ausführungen der kausalen Theorie des Handelns fällt es auf, dass der Unterschied zwischen konkret datierbaren Änderungen intentionaler Einstellungen und den intentionalen Gehalten, auf die sich diese Einstellungen beziehen, meistens nur unscharf markiert wird. Davidson schreibt:

Typically, in fact, the connection between an agent's having certain attitudes and his acting is closer than these remarks suggest, for it is changes in the attitudes, which are events, and which are often unmentioned causes. (...) And we can often turn a causal explanation which mentions beliefs and desires into an explanation which refers to an event or events by saying the cause of the action was the advent of one or both of the belief-desire pair. ${ }^{19}$

Dieses Zitat macht deutlich, wie Gründe zu Ursachen uminterpretiert werden. Nicht das, was geglaubt wird, oder das, was gewünscht wird, macht verständlich, warum die Handlung vollzogen worden ist. Es sind vielmehr Änderungen in den intentionalen Einstellungen des Handelnden, etwa die plötzliche Gewissheit, dass p, oder die Angst, dass p, bzw. das Aufkommen oder Abflauen eines Wunsches, die als Ursachen für die zu erklärende Handlung herangezogen werden. Diese Interpretation ist aber durchaus problematisch, wie im folgenden Beispiel deutlich wird: Wenn wir sagen, dass Josef endlich handelt, weil ihm der richtige Gedanke ,in den Kopf geschossen“ ist, so erklärt ein Verweis auf das plötzliche Auftauchen des entscheidenden Gedankens nicht, warum Josef so handelt, sondern nur warum Josef jetzt und nicht zu einem anderen Zeitpunkt so handelt. Das Auftauchen des entscheidenden Handlungsgrundes vermag den Zeitpunkt des Handlungsvollzugs erklären. Es bleibt aber der intentionale Gehalt des jeweiligen Grundes, der erklärt, warum diese und nicht eine andere Handlung vollzogen worden ist bzw. warum genau diese Handlung aus der Sicht des Handelnden zu tun war.

19 Davidson (1993), 288. 
Der Übergang von beliefs und desires i.S. intentionaler Gehalte zu changes intentionaler Einstellungen oder advents neuer beliefs und desires ist nicht ohne weiteres gerechtfertigt. Erleichtert wird dieser Übergang durch den unterschiedlichen Gebrauch der Ausdrücke belief und desire. Zum einen können mit beliefs und desires intentionale Zustände oder Einstellungen gemeint sein, etwa der Zustand sich vor etwas zu fürchten, sich heftig etwas zu wünschen oder der Zustand des Überzeugtseins. Zum anderen kann man sich mit diesen Ausdrücken auf den Gehalt dieser intentionalen Einstellungen beziehen, d.h. auf das, wovon man überzeugt ist, bzw. auf das, was man sich wünscht. In einer Passage aus John Bishops Natural Agency wird der nicht leicht zu bemerkende Übergang von der einen Verwendungsweise zur anderen besonders deutlich:

Actions typically occur because their agents had reasons for performing them, and these reasons, it seems consist in sets of mental states - beliefs, desires, intentions, and the like. Thus, CTA [the causal theory of action, Anmerkung G. Gasser] proposes that an action consists in an event (or events) with a special kind of mental event-causal history $\left[\ldots .{ }^{20}\right.$

Während Bishop sich zu Beginn mit reasons noch auf den intentionalen Gehalt zu beziehen scheint, geht er im restlichen Abschnitt dazu über, reasons mit intentionalen Vorgängen im Handelnden bzw. Einstellungen des Handelnden gleichzusetzen. Eine solche Gleichsetzung ist aber nicht ohne weiteres gerechtfertigt. Intentionale Einstellungen sind in stärkerer oder schwächerer Form gegeben. Sie tauchen in verschiedener Intensität und Stärke auf und können durch den Wechsel der Aufmerksamkeit unterbrochen werden bzw. ganz aufhören. Gründe scheinen solche Eigenschaften nicht aufzuweisen. Die Art und Weise, wie ich mich auf einen Grund beziehe, kann sich natürlich ändern, aber der intentionale Gehalt des Grundes bleibt von solchen Änderungen psychischer Einstellungen unbetroffen: Ich kann felsenfest davon überzeugt sein, dass $\mathrm{X}$ der Fall ist; ich kann nur vermuten, dass $\mathrm{X}$ der Fall ist oder ich kann mit relativer Gewissheit annehmen, dass $\mathrm{X}$ der Fall ist. $\mathrm{Zu}$ verschiedenen Zeitpunkten kann ich mich in unterschiedlichen Einstellungen $\mathrm{zu}$ einem intentionalen Inhalt befinden. Der intentionale Gehalt selbst ändert sich dadurch aber nicht. Es ist naheliegend, Gründe für Handlungen mit dem

20 Zitiert nach Meixner (2001), 351. 
intentionalen Gehalt zu identifizieren, nicht mit möglichen intentionalen Einstellungen, die wir gegenüber dem intentionalen Gehalt einnehmen können. Intentionale Einstellungen mögen die konkrete Ausführung der Handlung beeinflussen, aber der Grund für die Handlung bleibt in den jeweiligen Fällen derselbe. Wer gewiss ist, dass $\mathrm{X}$ zum Ziel führt, der handelt schnell und selbstsicher, wer hingegen unsicher ist, vorsichtig und zögerlich; aber trotz der unterschiedlichen Handlungsausführung ist der Grund für die Handlung derselbe, nämlich der Inhalt der Überzeugung, dass X zum Ziel führt.

Angesichts dieses Befundes stellt sich die Frage, wie die kausale Handlungstheorie vorgehen soll. Wenn Gründe Ursachen sind, so müssen Gründe Teile von Kausalbeziehungen sein. Die einzige mögliche Lösung scheint darin zu liegen, die Unterscheidung zwischen dem intentionalen Gehalt, auf den man sich auf unterschiedliche Weise beziehen kann, und den Veränderungen intentionaler Einstellungen zugunsten einer Theorie aufzugeben, der zufolge nicht die Gehalte, auf die sich intentionale Einstellungen beziehen, für das Verstehen von Handlungen relevant sind, sondern die intentionalen Einstellungen selbst bzw. Änderungen derselben. Nur intentionale Einstellungen i.S. eines Vorgangs im Handelnden können stricto sensu Ursachen einer entsprechenden Handlung sein. In Ansätzen der kausalen Theorie des Handelns ist, soweit ich sehen kann, allerdings nicht allzu viel argumentative Arbeit geleistet worden, warum die Unterscheidung zwischen intentionalen Gehalten und intentionalen Einstellungen vernachlässigt werden kann. Argumentationsarbeit hierfür wäre aber notwendig, um den Eindruck zu beseitigen, dass es sich um eine mögliche Schwachstelle handelt, welche die kausale Theorie des Handelns übergeht, indem sie rasch und unauffällig zwischen verschiedenen Verwendungsweisen von reasons, beliefs, desires und attitudes hin- und herwechselt.

2.2. „Bloße Gründe“ und „wirksame Gründe“ in der kausalen Theorie des Handelns

Wenn Gründe Ursachen sind, so handelt es sich um „wirksame Gründe“: Wie wir bereits gesehen haben, ist das Standard-Argument der kausalen Theorie des Handelns ein Argument für die kausale Wirksamkeit von 
Gründen: Es ist plausibel anzunehmen, dass ein Grund nur dann eine Handlung erklären kann, wenn er nicht nur inhaltlich zur Handlung „passt“, sondern auch kausal wirksam im Hervorbringen der Handlung war. $^{21}$

Dieses Argument operiert mit einer sogenannten kontrastiven WarumFrage. Kontrastive Warum-Fragen fragen danach, warum X im Gegensatz $z u \mathrm{Y}$ der Fall ist bzw. warum $\mathrm{X}$ im Gegensatz zu Y die vorliegende Tatsache erklärt. Um solche Fälle, in denen es darum geht, einen Kontrast zu erklären, adäquat zu begreifen, genügt es nicht, nur die eingetretene Tatsache alleine zu erklären. Vielmehr muss erklärt werden, warum gerade diese Tatsache eingetreten ist und nicht eine der anderen ebenfalls möglichen Alternativen. ${ }^{22}$

Kontrastive Warum-Fragen sind dann zufriedenstellend beantwortet, wenn aufgezeigt wird, was die Tatsache von ihren vermeintlichen Alternativen unterscheidet, d.h. was dazu geführt hat, dass aus den möglichen Alternativen eine zur Tatsache wurde und die andere eine bloße Möglichkeit blieb. Die Antwort der kausalen Theorie des Handelns auf die Frage, warum jemand aufgrund von $\mathrm{X}$ und nicht aufgrund von $\mathrm{Y}$ handelt, wo doch $\mathrm{X}$ und $\mathrm{Y}$ als Gründe zur Auswahl stehen, bleibt dürftig: Eigentlich wird nur darauf verwiesen, dass diese Frage am besten damit beantwortet wird, dass $\mathrm{X}$ eben auch die Ursache der Handlung war, während Y nicht den Status der Ursache für sich in Anspruch nehmen kann. Damit ist obige Frage aber keineswegs beantwortet. Wir wollen ja wissen, warum X im Gegensatz zu Y Ursache der Handlung war, in Anbetracht der Tatsache, dass $\mathrm{X}$ und $\mathrm{Y}$ als respektable Gründe für die Handlung in Frage kommen. Der Verweis auf den kausalen Status von X im Gegensatz zu Y hat eher den Anschein einer Vertröstung als den einer zufriedenstellenden Antwort. Was wir wissen wollen ist nicht, dass Y keine Rolle in der Ätiologie der Handlung spielt, sondern warum Y keine

21 In der Literatur wird dieses Argument auch als „Davidon’s Challenge“ bezeichnet. Siehe Mele (2003), 38.

22 Lipton (1991), 38: "One reason why explaining a contrast is sometimes harder than explaining the fact alone is that explaining a contrast requires giving causal information to distinguish the fact from the foil, and information that we accept as an explanation of the fact alone may not do this." 
Rolle spielt - angesichts der Tatsache, dass $\mathrm{Y}$ so wie $\mathrm{X}$ einen möglichen Handlungsgrund darstellt und ebenso wie $X$ auch dem Handelnden zugänglich ist. ${ }^{23}$

Für die kausale Theorie des Handelns stellt sich die Frage, wie mit nicht handlungswirksamen Gründen umzugehen ist: Warum werden diese Gründe nicht handlungswirksam, obwohl sie ante actum allem Anschein nach einen genauso vernünftigen Handlungsgrund darstellen? Worin besteht der entscheidende Unterschied zwischen Gründen als Ursachen und Gründen, die nicht kausal wirksam werden? Die kausale Theorie des Handelns kann diese Frage nicht einfach durch den Verweis beantworten, dass diese und nicht andere mögliche Gründe die entscheidenden Gründe für die zur Debatte stehende Handlung waren, weil ansonsten nicht diese, sondern eine andere Handlung verursacht worden wäre. Vielmehr gilt es aufzuzeigen, woran sich der Unterschied zwischen wirksamen und nicht wirksamen Gründen festmachen lässt.

\subsection{A-Personale Gründe}

Das Problem, wie nicht wirksame Gründe im Rahmen der kausalen Theorie des Handelns zu bestimmen sind, weist darauf hin, dass durch die Identifikation von wirksamen Gründen mit Ursachen die Frage virulent wird, ob die Rede von Gründen schlussendlich nicht gänzlich durch die Rede über Ursachen ersetzt werden kann. Gründe zeichnen sich dadurch aus, dass sie einem Handelnden zugänglich sein müssen. Intentionale Gehalte verweisen auf jemanden, der einen Zugang zu diesen intentionalen Gehalten hat. Wenn Gründe und Handlungen aber durch eine Kausalbeziehung aufeinander bezogen sind, so genügt es prinzipiell, wie bei anderen Ereignisfolgen auch, die kausalen Umstände und ihre Wirkmechanismen zu kennen, um zu wissen, warum dieses Ereignis eingetreten ist.

Der Fokus auf die Kausalrelationen droht die Perspektive des Handelnden für das Verstehen von Handlungen überflüssig zu machen.

23 Dickenson (2007), 15: „How do we make sense of cases in which an agent has reasons that are non-efficacious (not causes)? What's needed is an explanation that explicitly takes into account how a reason can be present in an agent and yet not be efficacious in producing the action." 
Kausale und intentionale Beziehungen schließen einander insofern aus, als kausale Vorgänge vom objektiven Standpunkt aus begriffen werden können, während intentionale Beziehungen auf die Perspektive des Handelnden angewiesen sind. Wir erhalten nur über die subjektive Perspektive des Handelnden einen Zugang zu seinen möglichen Handlungsgründen. Dies gilt aber nicht für Ursachen: Ursachen können, sie müssen aber nicht, dem Handelnden zugänglich sein. ${ }^{24}$

Eine rational vollzogene Handlung setzt voraus, dass der Handelnde im Lichte seiner Handlungsgründe eine Handlung möglichen Alternativen vorzieht. Wenn eine Handlung aber verursacht ist, so scheint sich die Frage nach der Auswahl zu erübrigen: Liegen entsprechende Bedingungen vor, so tritt die Wirkung ein. Zwischen Gründen als Inhalten, die dem Handelnden zugänglich sind und im Lichte derer eine Handlung vollzogen wird, und Ursachen als auslösenden Faktoren, die nicht an eine bestimmte subjektive Perspektive gebunden, sondern prinzipiell für jeden zugänglich sind, scheint eine Unverträglichkeit zu herrschen, die nicht aufgelöst werden kann. Die Rede von Bewusstsein, Gründen und Handlungen ist an eine Erste-Person-Perspektive ${ }^{25}$ geknüpft, die Rede von Ursachen und Kausalbeziehung hingegen ist es nicht. In einer Ontologie, welche die Erste Person berücksichtigt, wird der Handelnde als Abwägender von Gründen vorausgesetzt, der im Lichte dieser Gründe entscheidet und handelt. Wer bzw. was kommt als Träger von Gründen in Frage, wenn die Frage nach Gründen innerhalb einer Theorie der Ursachen verhandelt wird? Es legt sich wohl nahe, Gründe als Bestandteile neurophysiologischer Abläufe im Gehirn eines Lebewesens zu konzipieren.

Träger von Gründen im eigentlichen Sinne gibt es in diesem Modell nicht mehr, da Gründe selbst zu einem Teilaspekt komplexer

24 Kim (1998), 78: "For when you deliberate, you must call on what you want and believe about the world - your preferences and information - from your internal perspective, and that's the only thing you can call on. [...] Reasons for action, therefore, are necessarily internal reasons, reasons that are cognitively accessible to the agent. That is one crucial respect in which reasons for action differ from causes of actions: reasons must, but causes need not, be accessible to the agent." 
neurophysiologischer Abläufe werden. Letzten Endes landen wir bei komplexen Beschreibungen neurophysiologischer Abläufe, die verschiedene Aspekte und Funktionen aufweisen. Al Mele gibt unumwunden zu:

In cases of overt action what is triggered is obviously a physical process; and the triggering intentions, consequently, are realized in physical states - or so, at least someone with my philosophical prejudices would content. For this reason, a fully detailed answer to the question how, in a particular human being, the acquisition of a particular proximal intention triggers a particular set of actional mechanism capable of issuing in overt action will properly be cast (at least partly) in the language of neuro-physiology (or perhaps physics). ${ }^{26}$

Falls ich Mele an dieser Stelle richtig lese, so vertritt er die Ansicht, dass die Neurophysiologie (oder gar die Physik) das letzte Wort in der Erklärung menschlichen Handelns $\mathrm{zu}$ sprechen hat. Uns vertrauten Handlungserklärungen scheint der Status der Vorläufigkeit anzuhaften sie sind solange brauchbar, bis die ,hard sciences" das Instrumentarium und Vokabular entwickelt haben, um menschliches Handeln vollständig als neurophysiologischen bzw. physischen Vorgang beschreiben zu können. Die Stoßrichtung ist in einer solchen Argumentation klar vorgegeben: Die Rede von Personen, die Gründe abwägen und im Lichte dieser Gründe handeln, scheint zwar in der Umgangssprache von Relevanz zu sein. Wenn wir aber genau wissen wollen, wie Handlungen vorbereitet, realisiert und ausgeführt werden, so sollten wir nicht auf der Ebene des Handelnden und seiner Perspektive suchen, sondern im subpersonalen Bereich neurophysiologischer Mechanismen.

Diesen Eindruck bestätigt auch Elisabeth Pacherie, welche die kausale Theorie des Handelns mit empirischen Befunden zu ergänzen versucht. Dabei entwirft sie ein facettenreiches und in weiten Teilen plausibles Bild, welche Regulationsmechanismen und Voraussetzungen erfüllt sein müssen, damit wir handeln können. ${ }^{27}$ In eine ähnliche Richtung wie Mele weisend, schreibt sie:

[...] in a naturalistic non-dualistic framework, personal-level mental states are constituted or realized by complex physical states and a personal-level account of behavior must be backed up by a subpersonal explanation of how mental

26 Mele (1992), 178.

27 Siehe z.B. Pacherie (2000) und (2006). 
causation works. Subpersonal and personal-level explanations are pitched at different levels of generality and should therefore be seen as complementary rather than mutually exclusive. [kursiv G.G.] ${ }^{28}$

Pacherie weist zwar darauf hin, dass die verschiedenen Erklärungsebenen aufeinander bezogen und nicht als einander ausschließend verstanden werden sollten. Aber die Frage stellt sich trotzdem, was es eigentlich heißt, dass ,personal-level mental state“ in komplexen physischen Zuständen realisiert werden. Es ist unstrittig, dass es Aufgabe der Wissenschaft ist, die komplexen physischen Zustände zu untersuchen, die menschliches Handeln ermöglichen. Legt die Untersuchung dieser physischen Mechanismen aber auch nahe anzunehmen, dass in solch präzisen und detailreichen subpersonalen Erklärungen die eigentlichen Erklärungen für das Verstehen von Handlungen zu suchen sind? Man kann sich nur schwer des Eindrucks entziehen, dass es der kausalen Theorie des Handelns zufolge letzten Endes der Neurophysiologie überlassen ist, zu erklären, was wirklich vor sich geht, wenn gehandelt wird. Ähnlich wie wir bereits bei Davidson gesehen haben, scheint die Rede von Handelnden, die im Lichte ihrer Gründe entscheiden, vom epistemisch-semantischen Standpunkt des menschlichen kognitiven Apparats aus gesehen zwar sinnvoll, aber unter ontologischer Rücksicht bedeutungslos zu sein.

Halten wir fest: Ich habe dafür argumentiert, dass die Akzeptanz der kausalen Theorie des Handelns in eine bestimmte Richtung weist. Es lässt sich wohl ohne zu übertreiben eine große Sympathie feststellen, Fragen danach, was Handlungen zu Handlungen macht und wie wir Handlungen erklären sollen, innerhalb eines Rahmens zu verhandeln, der eigentlich aus neuronalen Mechanismen, subpersonalen Kontrollsystemen und handlungssteuernden Reaktionsschemata besteht. Aus dem Zusammenwirken all dieser komplexen Prozesse, welche eine empirische Kognitionswissenschaft langsam zu entwirren beginnt, entsteht unsere subjektive Erfahrung ein Handelnder zu sein. Unser subjektiver Zugang zum Handeln ist wie die Spitze eines Eisberges, die aus dem Wasser ragt: Wir sehen die Spitze, wissen aber nicht, was sich darunter verbirgt und den Verlauf der Spitze im Wasser festlegt. Der eigentliche Teil bleibt unseren

28 Pacherie (2006), 160. 
Augen verborgen. Anders ausgedrückt: Die kausale Handlungstheorie kann keinen wirksamen Damm gegen die Gefahr errichten, dass Handlungserklärungen von einer personalen auf eine a- und subpersonale Ebene abrutschen, in der kognitive Mechanismen und neurologische Systeme als Ursachen unseres Handelns angeführt werden. Die Suche nach Ursachen scheint geradezu danach $\mathrm{zu}$ verlangen, die personale Ebene zugunsten tiefer liegender subpersonaler Ebenen zu verlassen. Letzten Endes sind es diese Ebenen, wo Ursachen ihre Wirkkraft entfalten und falls überhaupt, dann nur in abgeleiteter Weise, die Rede rechtfertigen, dass wir als handelnde Personen unsere Handlungen vollziehen. ${ }^{29}$

\subsection{Die Auflösung des Handelnden}

Stricto sensu führt das Abrutschen von einer personalen auf eine subpersonale Ebene dazu, dass Handlungen in a-personale Vorgänge aufgelöst werden. Wenn Ereignisse Ursachen von Handlungen sind, so erübrigt es sich letzten Endes, auch noch den Handelnden für die Erklärung von Handlungen ins Spiel zu bringen. Handelnde werden vielmehr zu Orten, an denen Ursachen von Handlungen auftreten. Irving Thalberg bemerkte bereits vor geraumer Zeit:

More precisely, he [the agent, Anmerkung G.G.] is like an arena where 'his' calculations, his perceptual judgements, his noble and base inclinations, perhaps his repressed fantasies, his conscious terrors, rages, lusts and devotions, either contend or bend with each other. ${ }^{30}$

Nicht Handelnde qua Handelnde sind für die Erklärung menschlicher Handlungen interessant, sondern Handelnde insofern sie Orte sind, an denen wir Ursachen von Ereignisketten lokalisieren, die wir Handlungen nennen. Handelnde werden zu Bündeln möglicher Ursachen für Handlungen. Damit verschwindet letzten Endes der Handelnde qua Subjekt als unumgänglicher Referenzpunkt für das Verstehen von Handlungen. Diese Schlussfolgerung ist zweifelsohne problematisch, nicht nur für unser Alltagsverständnis als Handelnde, sondern auch weil i.e.S. nicht mehr zwischen Handlungen und natürlichen Vorgängen unterschieden werden kann. Etliche Kognitionswissenschaftler und Philosophen sind durchaus

29 Siehe dazu auch Baker (1993), 93.

30 Thalberg (1975/1980), 220. Siehe auch Velleman (2000), 123-129. 
bereit, diesen Preis zu zahlen. Im Grunde legt es die kausale Theorie des Handelns sogar nahe, diesen Preis zu zahlen. Wenn es ,agent-involving mental states and events ${ }^{631}$ sind, welche Handlungen verursachen und diese ontologisch gesprochen eigentlich physische Zustände und Ereignisse sind, dann erscheint die Frage nach dem Handelnden selbst als sekundär. J. D. Velleman hat diese Konsequenz klar gesehen. Er sieht das Problem, dass ,the causal role assigned to the agent by common sense reduces to, or supervenes on, causal relations among events and states of affairs. ‘32

Wie soll mit diesem Befund umgegangen werden? Für die kausale Theorie des Handelns gibt es nach meinem Dafürhalten zwei Möglichkeiten, wobei beide Deutungen die Rede von Personen als Handelnden stricto sensu aufgeben. Wer die Rede von handelnden Personen irgendwie in einem realistischen Sinn retten will, wird in Richtung eines „homunkularen Ansatzes“633 argumentieren, d.h. intentionale Fähigkeiten und Leistungen der handelnden Person werden auf natürliche Vorgänge innerhalb der Person zurückgeführt. Jemand kann mit gutem Recht als handelnde Person bezeichnet werden, da Systeme in dieser Person „Handlungs-Funktionen“ haben und diese „HandlungsFunktionen“ indirekt auf die Person selbst übertragen werden können schließlich befinden sich die Systeme ja in der Person. Velleman selbst entscheidet sich für eine homunkular angehauchte Position, indem er die Rede von handelnden Personen, wenigstens in einem indirekten Sinn, als zulässig ansieht, weil es handlungsinitiierende und - ausführende Systeme innerhalb der handelnden Person gibt. ${ }^{34}$

31 Ich übernehme die Redeweise von Schlosser (2008), 3.

32 Velleman (2000), 130.

$33 \mathrm{Zu}$ homunkularen Ansätzen in den Kognitionswissenschaften siehe Keil (2003).

34 Velleman (2000), 138: "A person is a fighter of infections and a digester of food in the sense that his parts include infection-fighting and food-digesting systems. Similarly, a person may be an initiator of actions - and hence an agent - in the sense that there is an action-initiating system within him, as system that performs the function in virtue of which he qualifies as an agent and which are ordinarily attributed to him in that capacity." 
Die zweite Möglichkeit besteht darin, die Rede von handelnden Personen in einem realistischen Sinn gänzlich fallen zu lassen und als Illusion anzusehen. Natürlich gibt es handlungsinitiierende und handlungsausführende Systeme, aber diese Systeme haben mit bewussten Entscheidungen und folglich auch mit Handlungen, so wie sie uns vom common sense her vertraut sind, nichts zu tun. Die Überzeugung, bewusst gehandelt zu haben, nimmt vielmehr die Rolle einer nachträglichen Interpretation eines Verhaltens als Handlung ein. So schreibt Jeannerod in Bezug auf Bewusstsein und Handeln:

The role of consciousness should rather be to ensure the continuity of subjective experience across actions which are - by necessity - executed automatically. Because it reads behavior rather than starting it, consciousness represents a background mechanism for the cognitive rearrangement after the action is completed, e.g. for justifying its results, or modifying the factors that have been at the origin of the movement if the latter turned out to be unsuccessful. ${ }^{35}$

Noch deutlicher bringt es Daniel Wegner auf den Punkt: Der eigentliche Grund, warum im Normalfall das, was wir tun, dem, was wir zu tun beabsichtigen, entspricht, liegt darin, dass wir über einen Mechanismus der Selbstzuschreibungen von Handlungen verfügen und nicht darin, dass wir als Handelnde wissen, was wir tun bzw. tun wollen. Handlungen unterscheiden sich von bloßen Prozessen der Natur nicht darin, dass Handlungen im Lichte von Überlegungen und Absichten eines Handelnden getroffen werden. Handlungen unterscheiden sich vielmehr darin, dass der menschliche Organismus über einen Selbstzuschreibungsmechanismus verfügt, der mit einem spezifischen Gefühl des Entscheidens bzw. Handelns einhergeht. Wegner schreibt:

These observations suggest that we feel conscious will as we perform our actions primarily in the case of actions that are caused by controlled processes. These processes allow us the conscious thoughts, self-observed actions, and time and attention necessary to draw causal influences about how our minds seem to be involved in producing our behaviors. In drawing these inferences, we accumulate the picture of a virtual agent, a mind that is apparently guiding the action. ${ }^{36}$

Wegners Position macht deutlich, wohin die kausale Theorie des Handelns letzten Endes führt: Wenn Gründe Ursachen sind, so gilt es im

35 Jeannerod (2006), 37.

36 Wegner (2005), 30. 
Hinblick auf eine angeblich empirisch fundierte Erklärung von Handlungen, die Rede von Gründen und sich frei entscheidenden Handelnden in eine Sprache kausaler Verursachung umzudeuten. Eine konsequente Weiterführung der kausalen Theorie des Handelns scheint sich derjenigen Begrifflichkeiten zu entledigen, die für das Verständnis menschlicher Handlungen von Nöten sind. Handelnde und ihre Gründe werden durch Systeme, Mechanismen und Ursachen ersetzt. Wenn wir uns als Handelnde begreifen, so ist dies letzten Endes die Folge bestimmter Mechanismen, aus deren Aktivität sich subjektiv das Gefühl, gehandelt zu haben, entwickelt. Die „ontologischen Taktangeber ${ }^{637}$ unseres Handelns sind aber sub-personale Prozesse und Zustände. In der Ontologie bleibt der Handelnde vollends auf der Strecke, in der Epistemologie bleibt dem vermeintlichen Handelnden nur die subjektive Vorstellung, ein Handelnder zu sein.

Falls diese Argumentation korrekt ist, so kann man innerhalb des Begriffs- und Erklärungsrahmens der kausalen Theorie des Handelns nicht mehr von freien Handelnden und bewusst vollzogenen Handlungen sprechen. Besser sollte man von „Quasi-Handelnden“, „QuasiEntscheidungen“" und "Quasi-Freiheit" sprechen $^{38}$, da wir uns ja nur subjektiv als frei Handelnde begreifen bzw. wir uns - konstruktivistisch formuliert - als solche entwerfen. ${ }^{39}$

\section{Schlussfolgerung}

Ich habe versucht, deutlich zu machen, wohin die kausale Theorie des Handelns führt. Das Hauptproblem der kausalen Theorie habe ich darin verortet, dass eine konsequente Anwendung des Gründe-sind-UrsachenAnsatzes Gründe durch Ursachen und Handelnde durch sub-personale Systeme und Mechanismen ersetzt. Eine konsequente Weiterführung der kausalen Theorie des Handelns kommt nicht umhin, die Rede von Gründen

37 Siehe (Wingert) 2006, 253.

38 Keil (2007), 79.

39 Mit einem solchen Konstruktivismus sympathisiert offensichtlich Walde (2006), 200-202. 
des Handelnden als Rede von Zuständen im Handelnden umzudeuten. Inwieweit die Rede von freien Entscheidungen in der Folge nicht in eine façon de parler aufgrund unserer kognitiven Bedürfnisse mündet, die eines fundamentum in re entbehrt, ist eine weitere Frage, welche die kausale Theorie des Handelns nicht umgehen kann.

Auf jeden Fall scheint unser Alltagsverständnis von Handlungen auf einer Ontologie zu fußen, in der frei Handelnde vorausgesetzt werden. Bereits Henrik von Wright machte sich dafür stark, dass unser Konzept des Handelns bzw. die Zuschreibung von Handlungen einen Begriffsrahmen impliziert, der die Freiheit des Handelnden voraussetzt. ${ }^{40}$ Das Problem der kausalen Theorie des Handelns liegt darin, dass kausale Beziehungen in der Welt die subjektive Perspektive des Handelnden nicht fassen können. Ebenso wenig kann aufgezeigt werden, dass selbstbestimmtes und freies Handeln die Wirkung einer bestimmten Ursache ist, die zu den Ursachen bei „natürlich“ ablaufenden Vorgängen noch hinzukommt. Selbstbewusstes und freies Handeln kann nicht durch „Ursachen der Freiheit" i.S. eines „Freiheitsaufweises“ angezeigt werden.

Daher stellt sich die Frage, ob ein Erklären, Deuten und Verstehen menschlichen Handelns über Kausalrelationen überhaupt möglich ist. Anhand der kausalen Theorie des Handelns habe ich dafür zu argumentieren versucht, dass dies nicht der Fall ist. Ich neige dazu anzunehmen, dass dies auf die sogenannten agenskausalen Varianten ebenso zutrifft, insofern in diesen Ansätzen neben Ereignissen nur ein zusätzlicher Kausalfaktor - der Handelnde selbst - ins Spiel gebracht wird. ${ }^{41}$ Es legt sich nahe zu überlegen, ob Ansätze in der Tradition der Handlungserklärungen durch Gründe nicht besser dafür geeignet sind, menschliches Handeln zu erklären, ${ }^{42}$ während kausale Ansätze für Erklärungen jener Vorgänge reserviert werden sollten, die keiner

40 Von Wright (1980), 78-79. Siehe auch Keil (2007), 78-80.

41 Siehe z.B. die Ansätze von O'Connor (1995 und 2000) und Clarke (1995 und 1996). So schreibt etwa Clarke (1995), 207: "Agent causation is a relation, the first relatum of which is an agent or person and the second relatum of which is an event. (...) The only difference between the two kinds of causation concerns the types of entities related, not the relation."

42 Siehe etwa neuerdings Nida-Rümelin (2005) und Hacker (2007), Kap. 6 und 7. 
Berücksichtigung einer Ontologie der Ersten Person bedürfen. Einen Versuch wert sind Überlegungen in diese Richtung angesichts der aufgezeigten Probleme allemal.

\section{Literatur}

Baker, L. R. (1993), Metaphysics and Mental Causation, in: Heil, J. / Mele, A., eds. (1993), Mental Causation. Oxford: Clarendon Press, 75-95.

Beckermann, A. (2006), Neuronale Determiniertheit und Freiheit, in: Köchy, K. / Stederoth, D., Hgg. (2006), Willensfreiheit als interdisziplinäres Problem. Freiburg / München: Verlag Karl Alber, 289-304.

Clarke, R. (1995), Toward a Credible Agent-Causal Account of Free Will, in: O'Connor, T., ed. (1995), Agents, Causes, and Events: Essays on Indeterminism and Free Will. New York: Oxford University Press, 201215.

Clarke, R. (1996), Agent Causation and Event Causation in the Production of Free Action, in: Philosophical Topics 24, no. 2, 19-48.

Davidson, D. (1963/21982), Actions, Reasons, and Causes, in: Davidson, D. Essays on Actions and Events. Oxford: Clarendon Press, 3-19.

Davidson, D. (1987/2006), Probleme der Handlungserklärung, in: Davidson, D. Probleme der Rationalität, übers. von Joachim Schulte. Frankfurt a. Main: Suhrkamp, 178-203.

Davidson, D. (1993), Reply to Peter Bieri, in: Stoecker, R., ed. (1993) Reflecting Davidson. Berlin / New York: Walter de Gruyter, 311-313.

Dickenson, J. (2007), Reasons, Causes and Contrasts, in: Pacific Philosophical Quarterly 88, 1-23.

Evnine, S. (1991), Donald Davidson. Oxford: Polity Press.

Habermas, J. (2007), Das Sprachspiel verantwortlicher Urheberschaft und das Problem der Willensfreiheit: Wie lässt sich der epistemische Dualismus mit einem ontologischen Monismus versöhnen?, in: Krüger, H.P., Hg. (2007), Hirn als Subjekt. Philosophische Grenzfragen der Neurobiologie. Deutsche Zeitschrift für Philosophie, Sonderband 15. Berlin: Akademie Verlag, 267-304. 
Hacker, P. M.S. (2007), Human Nature: The Categorial Framework. Oxford: Blackwell Publishing.

Jeannerod, M. (2006), Consciousness of Action as an Embodied Consciousness, in: Pockett, S. u.a., eds. (2006), Does Consciousness Cause Behaviour? Cambridge, MA: MIT Press, 25-38.

Keil, G. (2000), Handeln und Verursachen. Frankfurt a. Main: Vittorio Klostermann.

Keil, G. (2003), Homunukulismus in den Kognitionswissenschaften, in: Köhler, W. und Mutschler, H.D., Hgg. (2003), Ist der Geist berechenbar? Darmstadt: Wissenschaftliche Buchgesellschaft.

Keil, G. (2007), Willensfreiheit. Berlin / New York: Walter de Gruyter.

Kim, J. (1998), Reasons and the First Person, in: Bransen, J. and Cuypers, S.E., eds. (1998), Human Action, Deliberation and Causation. Dordrecht: Kluwer Academic Publishers, 67-87.

Lanz, P. (1993), The Explanatory Force of Action Explanations, in: Stoecker, R., ed. (1993), Reflecting Davidson. Berlin / New York: Walter de Gruyter, 291-301.

Lipton, P. (1991), Inference to the best Explanation. London / New York: Routledge.

Mele, A. R. (1992), Springs of Action. Oxford: Oxford University Press.

Mele, A. R. (2003), Motivation and Agency. Oxford: Oxford University Press.

Meixner, U. (2001), Theorie der Kausalität. Ein Leitbegriff zum Kausalbegriff in zwei Teilen. Paderborn: Mentis.

Nida-Rümelin, Julia (2005), Über menschliche Freiheit. Stuttgart: Philipp Reclam.

O’Connor, T. (1995), Agent Causation, in: O’Connor, T., ed. (1995), Agents, Causes, and Events: Essays on Indeterminism and Free Will. New York: Oxford University Press, 173-200.

O'Connor, T. (2000), Persons and Causes: The Metaphysics of Free Will. New York: Oxford University Press.

Pacherie, E. (2000), The Content of Intentions, in: Mind \& Language 15, No. 4, (2000), 400-432. 
Pacherie, E. (2006), Toward a Dynamic Theory of Intentions, in: Pockett, S. u.a., eds. (2006), Does Consciousness Cause Behaviour? Cambridge, MA: MIT Press, 145-167.

Schlosser, M. (2008), Agent-Causation and Agential Control, in: Philosophical Explorations 11, 3-21.

Searle, J. R. (1987), Intentionalität. Eine Abhandlung zur Philosophie des Geistes. Frankfurt am Main: Suhrkamp.

Searle, J. R. (1991), Response: The Background of Intentionality and Action, in: Lepore, E. and Van Gulick, R., eds. (1991), John Searle and His Critics. Cambridge, MA: Basil Blackwell, 289-299.

Searle, J. R. (1992), The Rediscovery of the Mind. Cambridge, MA: MIT Press.

Searle J., R. (2001), Rationality in Action. Cambridge, MA: MIT Press.

Thalberg I. (1975), How does Agent Causality Work?, in: Brand, M. I Walton, D., eds. (1980), Action Theory. Dordrecht / Boston: D. Reidel Publishing Company, 213-238.

Velleman, D. (2000), What Happens When Someone Acts?, in: Velleman, D. The Possibility of Practical Reason. Oxford: Clarendon Press, 123143.

Walde, B. (2006), Willensfreiheit und Hirnforschung. Paderborn: Mentis. Wegner, D. M. (2005), Who Is the Controller of Controlled Processes?, in: Hassin, R.R. u.a., eds. (2005), The New Unconscious. Oxford: Oxford University Press, 19-36.

Wingert, L. (2006), Grenzen der naturalistischen Selbstobjektivierung, in: Sturma, D., Hg. (2006), Philosophie und Neurowissenschaften. Frankfurt a. Main: Suhrkamp, 240-260.

Wright, G. H. von (1980), Freedom and Determination, in: Acta Philosophica Fennica 31, 5-88. 